\title{
CONSUMO DE TABACO Y MORTALIDAD POR CÁNCER VESICAL EN ESPAÑA.
}

\author{
Daniel Santos Arrontes, Manuel Fernández Arjona, María Paz Valer López-Fando', Carlos \\ Pascual Mateo², Fernando De Castro Barbosa, Ignacio Cortés Aránguez e Ignacio Jiménez'.
}

Servicio de Urología. Hospital Santa Bárbara. Puertollano. Ciudad Real. Práctica privada y

Servicio de Urología². Hospital Universitario de Getafe. Madrid. España.

\begin{abstract}
Resumen.- OBJETIVO: Determinar la tendencia de la mortalidad ajustada por cáncer vesical y establecer una correlación con el consumo de tabaco en las diferentes Comunidades españolas a lo largo de un periodo de quince años.
\end{abstract}

MÉTODOS: Se evaluarán las tendencias de mortalidad asociada al cáncer vesical en el periodo comprendido entre el 1 de Enero de 1989 y el 31 de Diciembre de 2002, en el área geográfica de España, así como las tendencias en el consumo de tabaco durante el mismo periodo. Los datos demográficos y de mortalidad se obtuvieron del Instituto Nacional de Estadística y los datos de consumo de tabaco se obtuvieron del Comisionado para el Mercado de Tabacos del Ministerio de Economia y Hacienda. Se calcularán el número medio de cajetillas de cigarrillos ( 1 cajetilla equivale a 20 cigarrillos) consumidas por persona; así como las tasas de mortalidad ajustadas por cáncer de vejiga expresada como fallecimientos por dicha causa por 100.000 personas y año, y el ratio entre las cajetillas de cigarrillos consumidos y la tasa de mortalidad ajustada. Se determinará el incremento porcentual anual durante todo el periodo, tanto para la tasa de mortalidad como para el consumo de tabaco, y se evaluará la correlación entre ambas tendencias mediante el coeficiente de Spearman.

RESULTADOS: Durante todo el periodo a estudio, la tasa media de mortalidad ajustada por cáncer vesical fue de 9,4 fallecidos por cada 100,000 habitantes y año, y el consumo medio de tabaco fue de 109,7 cajetillas por persona y año. Se ha producido un incremento de la mortalidad asociada a cáncer vesical de un 2,05\% anual, mientras que el consumo de cigarrillos ha disminuido un 1,3\% al año. El test de Spearman no muestra correlación entre ambos factores ( $p=0,722)$.

CONCLUSIONES: A pesar de las medidas de prevención del tabaquismo en nuetro medio, continúa produciéndose un importante consumo del mismo. El incremento en la incidencia de cáncer vesical no parece estar en relación con un mayor consumo de tabaco en la actualidad, por lo que puede estar en relación con otros factores genéticos o ambientales.

Palabras clave: Neoplasias vesicales. Mortalidad. Tabaco.

Summary.- OBJECTIVES: To establish the trend of the bladder cancer adjusted mortality and its correlation with tobacco consumption in different Spanish Autonomic Communities over a 15 year period. 
METHODS: We evaluate the trends of mortality rates associated with bladder cancer between January 7 st 1989 and December 31 st 2002 in the geographic area of Spain, as well as the tendency of tobacco consumption. Demographic and mortality data were obtained from the National Institute of Statistics, and consumption data were obtained from the Commissioner for the Tobacco Market in the Spanish Economics and Finances Ministry. We calculate the average number of cigarettes (1 package $=20$ cigarettes) consumed per person; we also calculate bladder cancer adjusted mortality rates, presented as number of deaths for that cause per 100.000 persons year, and the ratio between number of packages of cigarettes consumed and adjusted mortality rate. We determine the yearly percentage increase over the whole period of study for both the mortality rate and tobacco consumption, and evaluate their correlation by the Spearman's coefficient.

RESULTS: Mean adjusted bladder cancer mortality rate over the period of study was 9.4 deaths per 100.000 habitants year, and mean tobacco consumption was 109.7 packages per person year. There has been a $2.05 \%$ yearly increase of bladder cancer associated mortality, mean cigarette consumption has diminished 1.3\% per year. The Spearman's test did not show any correlation between both factors ( $p=0.722$ ).

CONCLUSIONS: Despite preventive measures for tobacco addiction in our country, there is an important continuous consumption. The increase in the incidence of bladder cancer does not seem to be related with higher tobacco consumption currently, therefore it may be related with other genetic or environmental factors.

Keywords: Bladder neoplasia. Mortality. Tobacco.

\section{INTRODUCCIÓN}

El cáncer vesical es una causa significativa de morbilidad y mortalidad. En España, en el año 2002 fallecieron 4197 pacientes constituyendo el $4,3 \%$ de la mortalidad oncológica en nuestro medio (1), siendo el cuarto tumor que asocia una mayor mortalidad en el varón -tras los cánceres de pulmón, próstata y colon-, y el octavo en la mujer (2).

Su desarrollo está relacionado con una serie de factores genéticos y ambientales. Dentro de estos últimos, el consumo de tabaco constituye un elemento etiopatogénico de considerable importancia, estando aumentado el riesgo de desarrollo hasta en cuatro veces en los pacientes fumadores; a su vez, este riesgo está relacionado con la cantidad de cigarrillos consumidos, la duración de la addicción al tabaco y el grado de inhalación de humo (3). Se cree que la tercera parte de los tumores vesicales están relacionados con el consumo de tabaco (4) y además en estos casos es mayor la probabilidad de afectación de la pared muscular en tumores de nuevo diagnóstico, que requieren un tratamiento más agresivo y tienen un peor pronóstico (5).

El objetivo de este estudio es determinar la tendencia de la mortalidad ajustada (estandarizada) del cáncer vesical y establecer una correlación con el consumo de tabaco en las diferentes Comunidades españolas a lo largo de un periodo de quince años.

\section{MATERIAL Y MÉTODOS}

En este estudio de tipo ecológico se evaluarán las tendencias de mortalidad asociada al cáncer vesical en el período comprendido entre el 1 de Enero de 1989 y el 31 de Diciembre de 2002, en el área geográfica de España 116 Comunidades Autónomas- CA-, 43,000,000 habitantes). Así como las tendencias en el consumo de tabaco durante el mismo periodo.

Los datos demográficos y de mortalidad se obtuvieron del Instituto Nacional de Estadística (INE) (1) y los datos de consumo de tabaco se obtuvieron del Comisionado para el Mercado de Tabacos del Ministerio de Economia y Hacienda (6). No se incluyen los datos de Canarias, Ceuta y Melilla por falta de registros válidos.

Se calcularán el número medio de cajetillas de cigarrillos (1 cajetilla equivale a 20 cigarrillos) consumidas por persona; así como las tasas de mortalidad ajustadas por cáncer de vejiga expresada como fallecimientos por dicha causa por 100.000 personas y año, y el ratio entre las cajetillas de cigarrillos consumidos y la tasa de mortalidad ajustada.

Se determinará el incremento porcentual anual durante todo el periodo, tanto para la tasa de mortalidad como para el consumo de tabaco, y se evaluará la correlación entre ambas tendencias mediante el coeficiente de Spearman.

Para el estudio estadístico se utilizará el software SPSS v12.0 para Windows, considerándose significativo una verosimilitud de la hipótesis nula inferior al 0,05. 


\section{RESULTADOS}

Los resultados de mortalidad y consumo de tabaco quedan registrados en la Tabla 1. Durante todo el periodo a estudio, la tasa media de mortalidad ajustada por cáncer vesical fue de 9,4 fallecidos por cada 100,000 habitantes y año, y el consumo medio de tabaco fue de 109,7 cajetillas por persona

\section{TABLA I. REGISTRO DE CONSUMO DE TABACO Y TASAS DE MORTALIDAD AJUSTADA ANUALES}

\begin{tabular}{|c|c|c|c|}
\hline AÑO & Cajetillas* & Tasa** & Ratio*** \\
\hline \multirow[t]{2}{*}{1989} & 105,5 & 8,1 & 13,4 \\
\hline & 20,9 & 1,3 & 3,9 \\
\hline \multirow[t]{2}{*}{1990} & 108,6 & 8,1 & 13,6 \\
\hline & 18,5 & 1,3 & 2,9 \\
\hline \multirow[t]{2}{*}{1991} & 114,8 & 8,7 & 13,4 \\
\hline & 20,7 & 1,5 & 3,0 \\
\hline \multirow[t]{2}{*}{1992} & 108,5 & 9,1 & 12,1 \\
\hline & 20,3 & 1,2 & 2,6 \\
\hline \multirow[t]{2}{*}{1993} & 99,8 & 9,3 & 11,0 \\
\hline & 19,6 & 1,1 & 3,1 \\
\hline \multirow[t]{2}{*}{1994} & 107,8 & 9,1 & 12,0 \\
\hline & 20,4 & 1,1 & 2,6 \\
\hline \multirow[t]{2}{*}{1995} & 100,8 & 9,2 & 11,3 \\
\hline & 20,5 & 1,7 & 3,4 \\
\hline \multirow[t]{2}{*}{1996} & 98,2 & 9,3 & 10,9 \\
\hline & 22,7 & 1,5 & 3,3 \\
\hline \multirow[t]{2}{*}{1998} & 116,6 & 9,8 & 12,2 \\
\hline & 21,5 & 1,3 & 3,0 \\
\hline \multirow[t]{2}{*}{1999} & 115,0 & 10,4 & 11,3 \\
\hline & 26,2 & 1,3 & 3,6 \\
\hline \multirow[t]{2}{*}{2000} & 116,6 & 10,4 & 11,6 \\
\hline & 30,4 & 1,6 & 4,1 \\
\hline \multirow[t]{2}{*}{2001} & 117,8 & 10,4 & 11,7 \\
\hline & 29,5 & 1,8 & 4,3 \\
\hline \multirow[t]{2}{*}{2002} & 115,7 & 10,8 & 11,1 \\
\hline & 27,2 & 1,8 & 3,6 \\
\hline \multirow[t]{2}{*}{ Total } & 109,7 & 9,4 & 12,0 \\
\hline & 23,6 & 1,6 & 3,4 \\
\hline
\end{tabular}

Cajetillas*: Número medio de cajetillas (20 cigarrillos) consumidos por persona y año. Tasas **: Tasa ajustada de mortalidad por cáncer vesical hallada de la media de la tasa ajustada de mortalidad para cada una de las provincias. Ratio ***: Cociente entre cajetillas * y Tasas **. El primer valor representa la media y el segundo la desviación estándar.

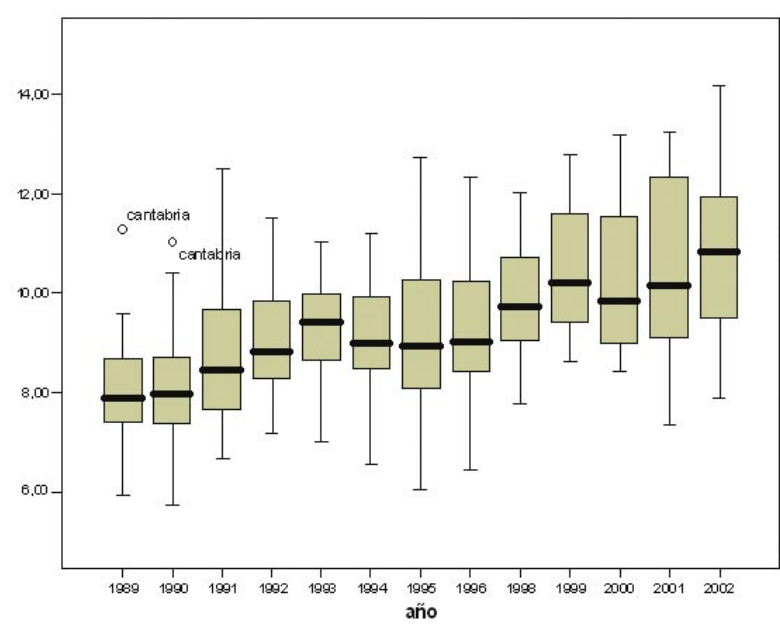

FIGURA 1. Representación gráfica de la tasa de mortalidad ajustada por año y CA.

y año, lo que equivale a 2194 cigarrillos/año, o lo que es lo mismo 6 cigarrillos/persona y día. Durante todo el periodo se ha producido un incremento de la mortalidad asociada a cáncer vesical de un 2,05 $\%$ anual, mientras que el consumo de cigarrillos ha disminuido un $1,3 \%$ al año.

En las Figuras 1 y 2 se representan la mortalidad cáncer específica y el consumo de tabaco, por año. Nótese que la tasa de mortalidad en Cantabria fue significativamente superior en los años 1989 y 1990, y que el consumo de tabaco es significativamente inferior en Galicia durante los primeros años del estudio, y reiteradamente superior en Baleares durante todo el periodo.

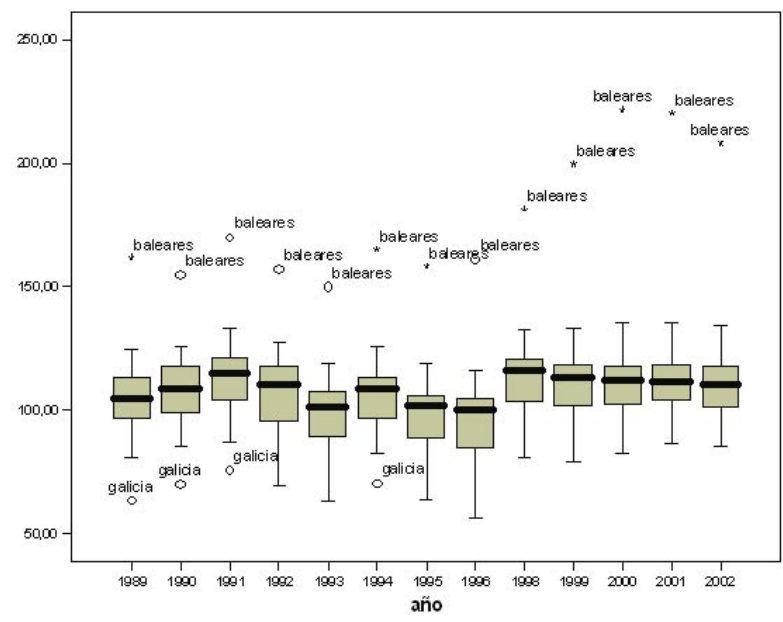

FIGURA 2. Consumo medio de cajetillas por año y CA. 
Las tasas de mortalidad y consumo anual quedan representadas en la Figura 3, donde se puede apreciar que en los últimos años se ha producido un "plateau" en el consumo de tabaco y al mismo tiempo un incremento de la mortalidad asociada a cáncer vesical. El test de Spearman no muestra correlación entre ambos factores $(p=0,722)$.

\section{DISCUSIÓN}

El consumo de tabaco constituye un problema de primer orden en nuestro país. España es el país de la Unión Europea (UE) con mayor índice de tabaquismo, con un $34,4 \%$ de fumadores habituales mayores de 16 años, según la Encuesta Nacional de Salud del Ministerio de Sanidad y Consumo (1). Además también ocupa el primer lugar de la UE en cuanto a número de mujeres jóvenes fumadoras. Se declaran consumidoras habituales de tabaco más de un $40 \%$ de las comprendidas entre los 15 y 25 años. En los últimos veinte años se ha multiplicado por tres

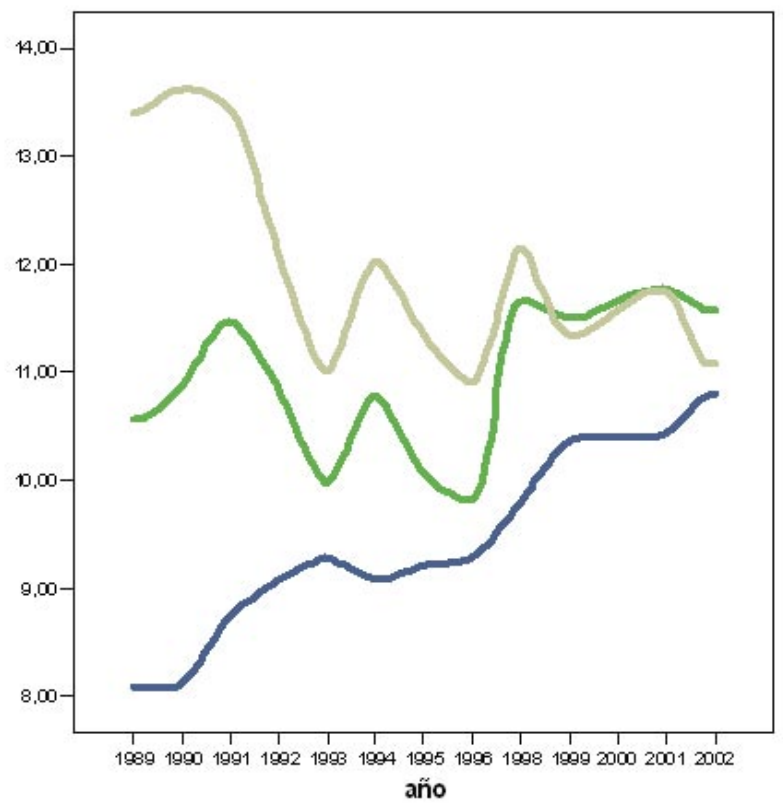

- Tasa de mortalidad — cajetillas por persona - ratio

FIGURA 3. Representación gráfica de la tasa de mortalidad ajustada, el consumo de cajetillas por persona y la ratio. Para adaptarlo a la escala los datos de consumo de cajetillas/persona y año están divididos entre 10. el porcentaje de mujeres fumadoras. Por todo ello es de preveer que a corto plazo el tabaco se convertirá en una de las principales causas de muerte por delante de enfermedades como el Sida o los accidentes de tráfico.

Actualmente una persona muere cada ocho segundos en el mundo a causa del tabaquismo, mientras que en España ya es la principal causa de enfermedad y de muerte evitable y unas 56.000 personas mueren cada año por su causa directa (7). En este estudio la media de consumo es superior a las 100 cajetillas por persona y año.

No obstante, todos los estudios epidemiológicos basados en tabaco presentan un problema de difícil solución, y es que desconocemos el periodo de latencia desde el inico de la adicción hasta el desarrollo del tumor, y desde el diagnóstico de este hasta que pueda o no causar el fallecimiento del individuo. Lógicamente esto supone un importante problema, pues es muy posible que los pacientes fallecidos hayan sido fumadores hace muchos años, con lo que realmente no tiene porque haber relación entre el consumo actual de tabaco y las tasas de mortalidad actual. De hecho hemos visto que no existe correlación, pero es posible que si la haya entre el consumo actual de tabaco y la mortalidad futura.

Por todo ello, es posible, que el incremento en la mortalidad por cáncer vesical que hemos detectado esté en relación con el incremento pasado en el consumo de tabaco. Esta teoría es sostenida por diferentes autores $(8,9)$ y además también justificaría el incremento en la incidencia y en la mortalidad en mujeres (10).

Otra limitación importante es que el estudio está basado en datos gubernamentales de venta al público de tabaco. Esto puede ser un problema pues los datos de venta no se tienen porque corresponder a los de consumo, pues parte de la venta podría ser secundaria a consumo por personas "en tránsito". De esta forma, el consumo en Baleares podría estar en relación con la venta masiva a turistas, que lógicamente fallecen en su país de origen.

Además de todo ello, los estudios epidemiológicos basado en certificados de defunción también estas sesgados, ya que muchas veces pueden omitirse enfermedades fundamentales como causa de muerte, no obstante, las estimaciones de mortalidad de la Organización Mundial de la Salud basadas en modelos matemáticos no se ajustan fielmente a la realidad. Por ejemplo, la tasa de mortalidad ajustada para el cáncer vesical en 1998 fue 6,56 fallecidos por 100,000 habitantes y año (11). 
Por último, el hecho de calcular la ratio nos permite evaluar ambas tendencias conjuntamente. Podemos apreciar en la figura 3, que se ha producido un descenso de la misma, en relación con el descenso del consumo y el aumento de la mortalidad. Sin embargo, esta tendencia parece tener una distribución cíclica durante los últimos años.

\section{CONCLUSIONES}

1. A pesar de las medidas de prevención del tabaquismo en nuetro medio, continúa produciéndose un importante consumo del mismo; la intensificación de estas medidas preventivas puede ser necesaria para disminuir la morbimortalidad asociada, sobre todo en las mujeres, donde es más esperable que se produzca un incremento de las mismas.

2. El incremento en la incidencia de cáncer vesical no parece estar en relación con un mayor consumo de tabaco en la actualidad, por lo que puede estar en relación con otros factores genéticos o ambientales. No obstante, el conocimiento de la historia natural del cáncer vesical asociado al tabaco aportará nuevos datos, que permitarán establecer previsiones fiables de incidencia y mortalidad.

\section{BIBLIOGRAFÍA y LECTURAS RECOMENDADAS ( ${ }^{*}$ lectura de interés $y^{* *}$ lectura fundamental)}

**1. http://www.ine.es. Última entrada 29/6/2005.

2. JEMAL, A.; TIWARI, R.C.; MURRAY, T. y cols.: "Cancer Statistics, 2004". CA Cancer J. Clin., 54: 8, 2004.

3. BURCH, J.D.; ROHAN, T.E.; HOWE, G.R. y cols.: "Risk of bladder cancer by source and type of tobacco exposure: A case control study". Int. J. Cancer., 44: 622, 1989.

4. HOWE G.R.; BURCH, J.D.; MILLER A.B. y cols.: "Tobacco use, occupation, coffee, various nutrients and bladder cancer'. J. Natl. Cancer Ins., 64: 701, 1980

5. FLESHNER, N.; GARLAND, J.; MOADEL, A. y cols.: "Influence of the smoking status on the disease related outcomes of patients with tobacco associated transitional cell carcinoma of the bladder". Cancer., 86: 2337, 1999.

*6. http:// www.cmtabacos.es. Última entrada 29/6/2005.

7. http://cajamadrid.lineasocial.com/pg041122/comunidad/cifras.htm. Última entrada 30/6/2005.

8. McLELLAN, R.A.; FRENCH, C.G.; BELL, D.G.: "Trends in the incidence of bladder cancer in Nova Scotia: a twenty-year perspective". Can. J. Urol., 10: 1880, 2003.

9. BORRAS, J.; BORRAS, J.M.; GALCERAN, J. y cols.: "Trends in smoking-related cancer incidence in Tarragona, Spain, 1980-96". Cancer Causes Control., 12: 903, 2001.

10. HAYNE, D.; ARYA, M.; QUINN, M.J. y cols.: "Currrent trends in bladder cancer in England and Wales". J. Urol., 172: 1051, 2004.

11. http://www-dep.iarc.fr/eucan/eucan.htm. Última entrada 30/6/2005. 